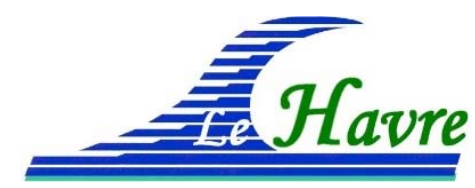

XVI èmes Journées Nationales Génie Côtier - Génie Civil

Le Havre, 2020

DOI:10.5150/jngcgc.2020.025 @ Editions Paralia CFL

disponible en ligne - http://www.paralia.fr - available online

\title{
Dynamique du maximum de turbidité dans le système estuarien du Wouri (Cameroun)
}

\section{Yannick FOSSI FOTSI ${ }^{1,2}$, Isabelle BRENON ${ }^{1}$, Nicolas POUVREAU ${ }^{3}$, Raphaël ONGUENE ${ }^{2}$, Yann FERRET ${ }^{3}$, Dieudonné DJOMBE ${ }^{4}$, Thibault COULOMBIER ${ }^{1}$, Jacques ETAME ${ }^{2}$}

\section{UMR 7266 LIENSs, Institut du Littoral et de l'Environnement, CNRS - Université} de La Rochelle, 17000 La Rochelle, France.

fossiyannick@gmail.com

2. Unité de formation doctorale de bio-géosciences et environnement, Université de Douala, BP 24157, Douala, Cameroun.

3. Shom-Service hydrographique et océanographique de la marine, 29200 Brest, France.

4. PAD, Port Autonome de Douala, BP 4020, Douala, Cameroun.

\section{Résumé :}

Les maxima de turbidité dans les estuaires influent sur le transport et le dépôt des sédiments fins, l'envasement des chenaux et la qualité de l'eau. La nécessité de compréhension et de prédiction de la dynamique des zones de turbidité maximale est donc un enjeu scientifique important et un besoin pour optimiser la gestion de ces eaux de transition. La dynamique sédimentaire estuarienne du Wouri (Cameroun) est fortement variable en fonction des saisons impactant les débits (de 250 à $3000 \mathrm{~m}^{3} / \mathrm{s}$ ). L'objectif de ce travail est l'analyse détaillée de mesures in situ pour comprendre cette dynamique dans la section fluviale de l'estuaire du Wouri. Pour se faire, deux sondes multi paramètres (OBS) ont été installées à $15 \mathrm{~km}$ de distance l'une de l'autre pour mesurer les caractéristiques physiques des eaux en amont et aval de l'estuaire. Au cours des 3 grandes saisons, la hauteur d'eau, la turbidité et la salinité ont été mesurées dans l'estuaire durant un mois en moyenne, afin de connaître l'impact de la variation tidale. A l'estuaire du Wouri, pendant les vives eaux, les contraintes de cisaillement sont plus fortes, ce qui favorise une plus forte remise en suspension des sédiments et donc de turbidité élevée. Pendant la saison de petite crue, l'observation persistante de la turbidité élevée permet de localiser le bouchon vaseux en aval de l'estuaire. Suivant les saisons, la remise en suspension des sédiments en amont de l'estuaire est contrôlée par les cycles dépôt/érosion dus à l'écoulement tidal.

Mots-clés :

Turbidité, Salinité, Estuaire du Wouri, Débit fluvial, Variation saisonnière 


\section{Thème 2 - Dynamique sédimentaire}

\section{Introduction}

La compréhension de l'hydrodynamique des estuaires représente un enjeu majeur, pour des raisons à la fois environnementales et socio-économiques. Ces environnements constituent généralement les zones privilégiées de construction des infrastructures portuaires et jouent un rôle essentiel dans les échanges économiques. De par leurs natures et morphologies qui ne cesse d'être modifiées, les estuaires sont soumis au phénomène de sédimentation. C'est le cas de l'estuaire du Wouri, qui abrite le Port Autonome de Douala (PAD). Le PAD est le principal port maritime du Cameroun et poumon économique de l'Afrique centrale. Il est situé à quelques $25 \mathrm{~km}$ de la mer sur le fleuve Wouri. Pour permettre l'entrée au port de la plupart des bateaux, un chenal d'accès est dragué en permanence car il est soumis à une forte sédimentation (DJOMBE SEPPO, 2011 ; FOSSI FOTSI, 2014). Comme tout estuaire mésotidal, l'estuaire du Wouri présente une zone de turbidité maximale (ZTM), appelée bouchon vaseux, à fortes concentrations en particules en suspension (MES) à la fois d'origine terrestre et marine (DJOMBE SEPPO, 2011 ; FOSSI FOTSI et al., 2019). La ZTM joue un rôle important dans le processus de sédimentation qui peuvent conduire à l'envasement des chenaux (ALLEN et al., 1980), affectant des profondeurs de navigation. La connaissance de la variabilité de la turbidité aux échelles de temps différentes est essentielle pour comprendre les processus hydro-sédimentaires, et la qualité des eaux dans cet estuaire camerounais. De nombreux travaux ont été publiés sur les phénomènes de turbidités maximales dans les estuaires du monde (GRABEMANN et al., 1997 ; MITCHELL et al., 2012 ; UNCLES et al., 1994). Les informations sur les estuaires africains restent très parcellaires et mal connues (CAPO et al., 2006). Le cas de l'estuaire du Wouri est très évocateur. A ce jour, et à notre connaissance, aucune étude n'a permis de comprendre les mécanismes de formation de la ZTM et ses dynamiques, l'essentiel des travaux ayant porté sur l'étude hydro sédimentaire des accès du PAD (ERECO EUROPEAN ECONOMIC, 1993 ; MIGNIOT, 1989 ; SOGREAH, 2006).

Cette étude vise à améliorer la quantification in-situ des caractéristiques du maximum de turbidité au cours des divers régimes fluviaux et de marée dans l'estuaire du Wouri et ainsi aboutir à une compréhension plus fine et approfondie de sa dynamique.

\section{Zone d'étude}

L'estuaire hyposynchrone du Wouri (FOSSI FOTSI et al., 2019) d'une superficie de 1 $200 \mathrm{~km}^{2}$, constitue une vaste zone humide, de $30 \mathrm{~km}$ de long et presque autant de large, ouverte sur l'océan Atlantique (NDONGO et al., 2015). Situé à $04^{\circ} 00^{\prime} 01^{\prime \prime}$ de latitude nord et $09^{\circ} 37^{\prime} 15^{\prime \prime}$ de longitude Est, il occupe le cœur du bassin sédimentaire de Douala (19000 km² dont $7000 \mathrm{~km}^{2}$ émergés). L'estuaire du Wouri, est caractérisé par des profondeurs plus élevées en aval ( $>8 \mathrm{~m}$ ) qu'en amont ( $<6 \mathrm{~m}$ ), (FOSSI FOTSI, 2014). 


\section{XVI'mes Journées Nationales Génie Côtier - Génie Civil \\ Le Havre 2020}

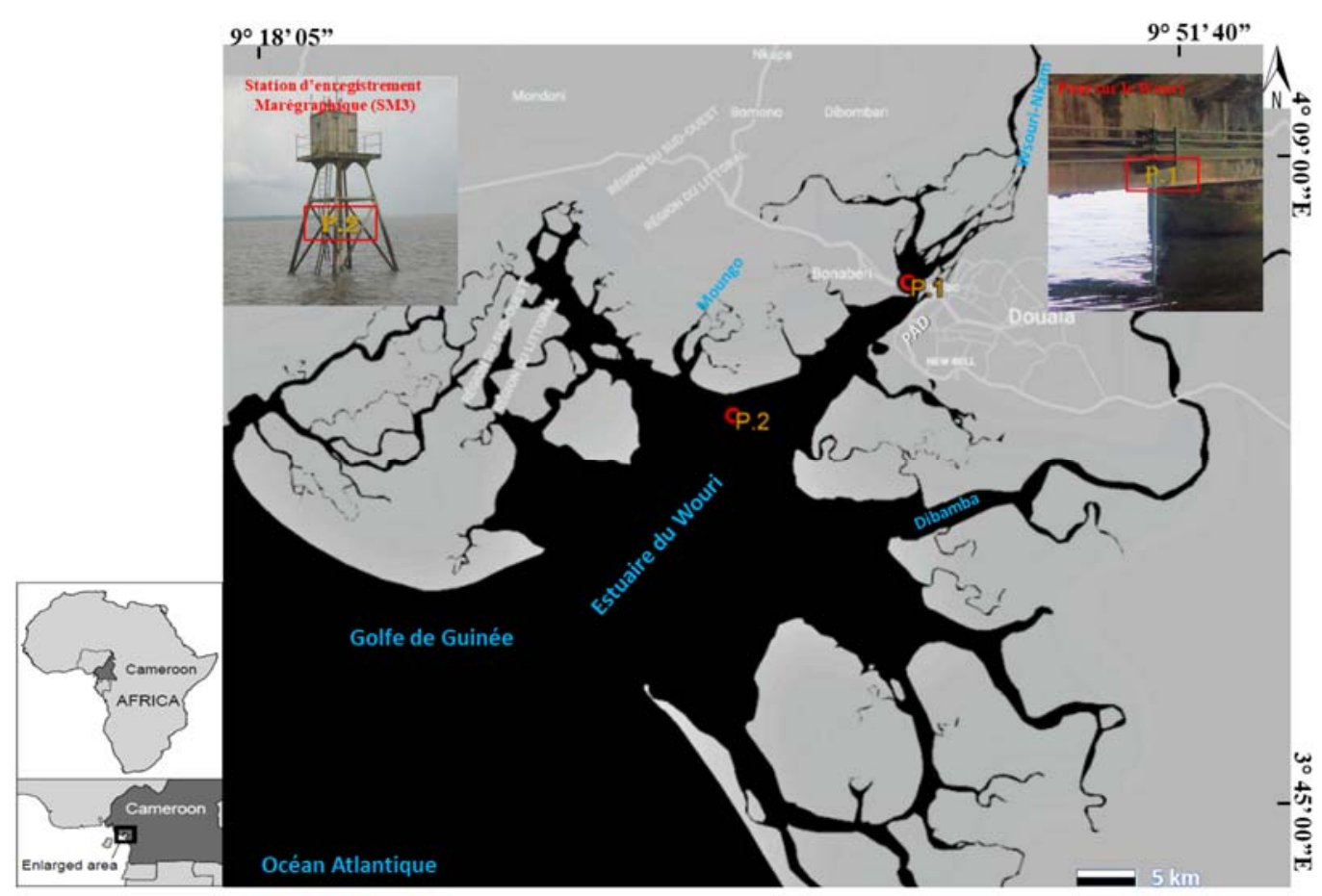

Figure 1. Carte de localisation de l'estuaire du Wouri montrant les deux stations de mesures des sondes multi-paramètres (P.1 et P.2).

Cette zone géographique est drainée par trois fleuves côtiers : la Dibamba, le Wouri et le Mungo (figure 1). Elle bénéficie d'un climat au régime équatorial d'un type particulier ou régime camerounais (DIN et al., 2008) avec une pluviométrie et débit annuels moyens d'égale valeur Si les précipitations et débits sont répartis sur toute l'année (figure 2), la longue saison humide de mars à novembre enregistre jusqu'à $94 \%$ de pluies et débits annuels (OLIVRY, 1974 ; TSALEFAC et al., 2003), alors que les mois secs (décembre à février) ne totalisent que $150 \mathrm{~mm}$ et $306 \mathrm{~m}^{3} / \mathrm{s}$, respectivement (AMELIE, 2018).

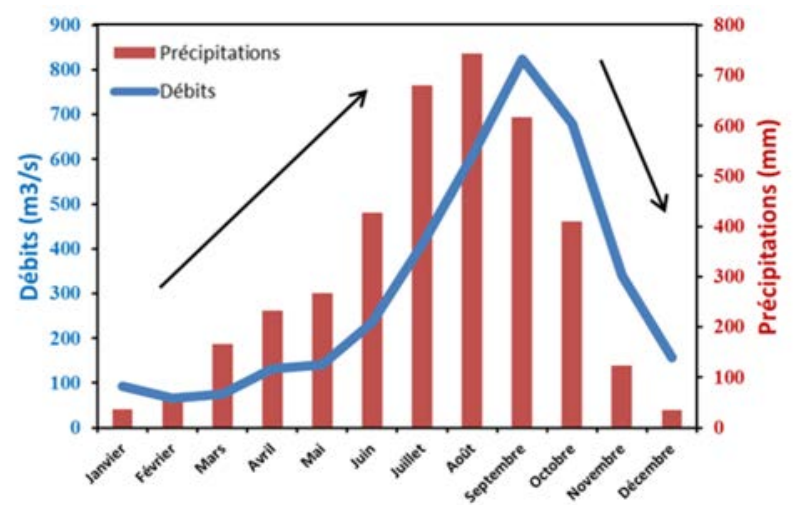

Figure 2. Précipitations mensuelles (année 2018) et débits moyens mensuels (période d'observation 1951-1974) à l'estuaire du Wouri. 


\section{Thème 2 - Dynamique sédimentaire}

L'amplitude moyenne de marée est de 2,5 m (FOSSI FOTSI, 2014). L'onde tidale qui se propage dans l'estuaire du Wouri est de type semi-diurne et tend à être déformée par la morphologie de l'estuaire, ce qui induit une asymétrie tidale forte (Fossi Fotsi, 2014).

Dans ce milieu, les différences d'altitude restent faibles, les reliefs dépassent rarement trois mètres, la végétation est une mangrove de palétuviers composée de Rhizophoras pouvant atteindre $40 \mathrm{~m}$ de hauteur et d'Avicennias qui colonisent les espaces intertidaux des terrains de sédimentation fine.

\section{Matériels et méthodes}

\subsection{Mesure in situ de hauteurs d'eau, de turbidité et de salinité}

Nous avons installé deux stations de mesure distantes de $15 \mathrm{~km}$ dans l'estuaire du Wouri : la station «Amont» $\mathrm{P} 1$, au niveau du pont sur le Wouri, et la station «Aval» $\mathrm{P} 2 \mathrm{au}$ niveau de la station marégraphique 3 présente dans l'estuaire (figure 1). Ces stations de mesures portent des sondes multi-paramètres (YSI 6600), équipées de capteurs qui assurent les mesures de hauteurs d'eau, de turbidité et de salinité, toutes les 5 minutes à $3 \mathrm{~m}$ sous la surface libre. Le capteur de turbidité mesure des valeurs entre 0 et 4000 NTU avec une précision de $10 \%$.

Les périodes de mesures ont été choisies pour permettre de caractériser l'influence des variations saisonnières de débits fluviaux (figure 2) : du 13 janvier au 27 février 2019 pour la saison d'étiage, du 8 mai au 7 juillet 2019 pour la petite crue, et du 22 juillet au 12 septembre 2019 pour la saison de grande crue. Lors de chaque campagne de mesures, la durée d'observation a permis de couvrir plusieurs cycles tidaux Morte-Eau (ME) Vive-Eau (VE) afin de pouvoir appréhender l'importance de la marée sur l'hydrodynamique sédimentaire de l'estuaire.

\subsection{Le prétraitement et analyse des données}

Lors de la période d'étiage, les eaux chaudes du Wouri sont propices à prolifération d'algues et de coquillages. Ce phénomène a eu pour effet de boucher le capteur de turbidité. Ainsi, avant toute analyse, une étape préalable de vérification et de nettoyage des données de turbidité a été indispensable. La valeur de turbidité de 4000 NTU correspond à la valeur de saturation du capteur, mais aussi à la valeur quand il est en défaut. Afin d'identifier et de supprimer les valeurs fausses, une appréciation visuelle a été associée à un critère additionnel développé pour distinguer les valeurs réellement aberrantes des valeurs caractéristiques d'une variation réaliste de turbidité. Le principe de ce test est de considérer comme fausses les valeurs égales à 4000 NTU lorsque les trois valeurs précédentes et suivantes sont inférieures à 1000 NTU c'est-à-dire quand il n'y a pas une augmentation progressive des valeurs jusqu'à 4000 NTU. 


\section{XVİ̀mes Journées Nationales Génie Côtier - Génie Civil \\ Le Havre 2020}

\section{Résultats}

4.1 Variabilité saisonnière des paramètres physiques dans l'estuaire du Wouri

Les variations saisonnières des paramètres hydrodynamiques (hauteurs d'eau, salinité et turbidité de l'eau) sont présentées à la figure 3 .

La majeure partie de l'année, le marnage observé en amont est plus important que celui mesuré en aval, variant respectivement en fonction du cycle ME-VE entre 1,2 $\mathrm{m}$ et 2,8 $\mathrm{m}$, et entre $1 \mathrm{~m}$ et $2,3 \mathrm{~m}$ (figures 3a1-3a2). Lors des grandes crues, cette différence diminue (de $0,4 \mathrm{~m}$ contre $0,07 \mathrm{~m}$ de différence) fortement, allant même jusqu'à s'inverser lors des pics de VE (figure $3 \mathrm{a} 3$ ).
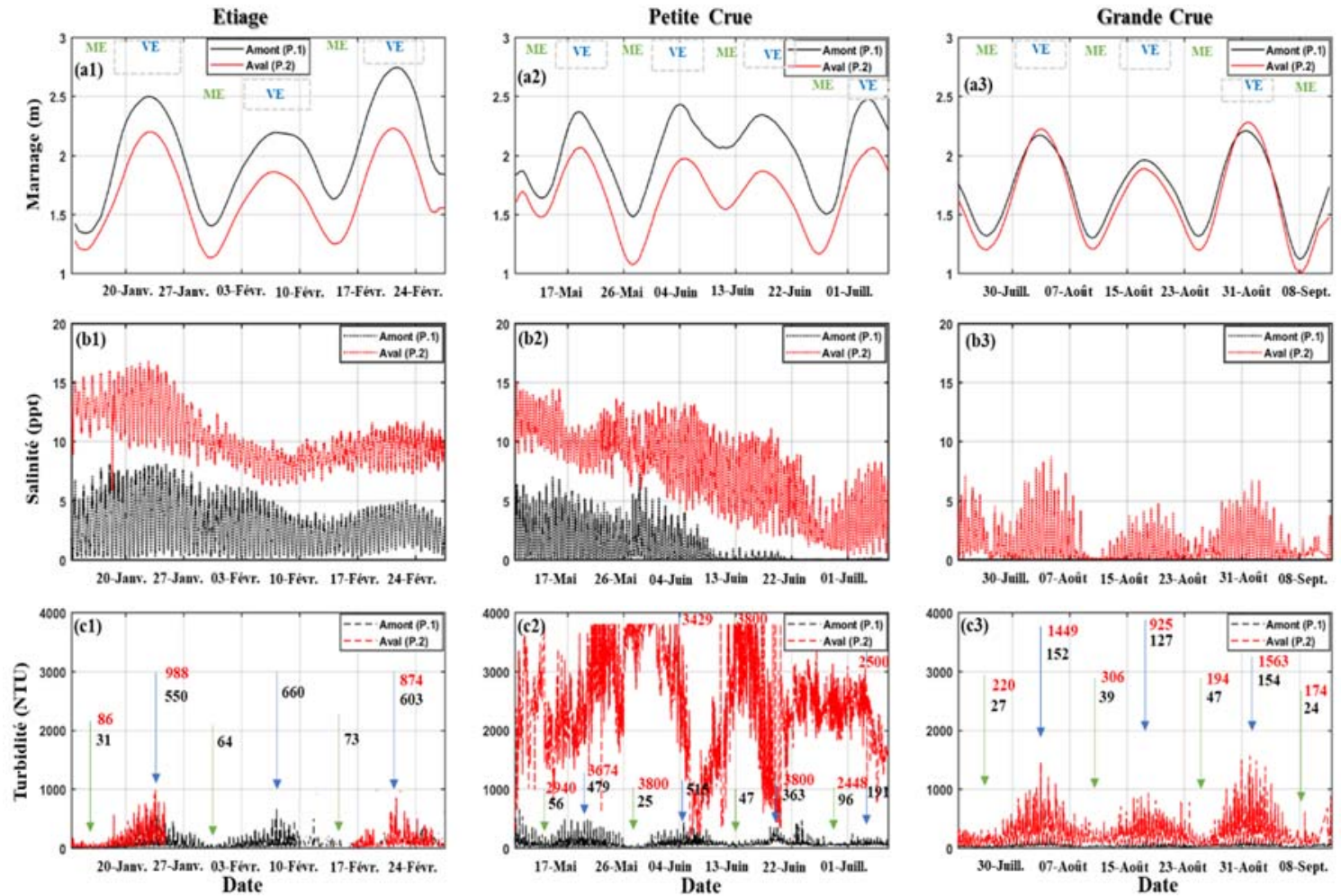

Figure 3. Séries chronologiques des niveaux de hauteurs de marée, illustrant les marées de vive et morte eau (a), de turbidité, présentant des pics de valeurs de turbidité de vive

et morte eau (b) et de salinité (c) aux deux stations de mesure (Amont et aval) à l'estuaire du Wouri en période d'étiage, de petite et grande crue.

Les variations de salinité observées (figure $4 \mathrm{~b}$ ) présentent des valeurs plus importantes en aval qu'en amont de l'estuaire. Elles montrent également une évolution décroissante de la saison d'étiage (sal._max.amont $=8,13$ et sal._max.aval $=16,83$ ), puis en petite crue ((sal._max.amont $=7,4$ et sal._max.aval $=15,02)$. Jusqu'à la grande crue (sal._max.amont $=0,04$ et sal._max.aval $=8,72)$ enregistrant des valeurs presque nulles en amont de l'estuaire 


\section{Thème 2 - Dynamique sédimentaire}

Pour la dynamique des matières en suspension, la turbidité mesurée est toujours supérieure en aval (station P2) par rapport à l'amont (station P1) (figure 3c). Les variations de turbidité observées sont globalement typiques d'une dynamique contrôlée par la marée : les valeurs de turbidité sont plus élevées en période de VE et les cyclicités semi-diurnes sont clairement visibles. Seules les mesures réalisées en aval de l'estuaire en petite crue (figure $3 \mathrm{c} 2$ ) présentent des variations qui semblent non corrélées à la marée (PM/BM et ME/VE), avec des valeurs très élevées (jusqu'à 3800NTU) la majeure partie du temps.

\subsection{Variations des turbidité, hauteurs d'eau, et salinité en fonction des cycles tidaux}

\subsubsection{Station Amont (P.1)}

En amont, les trois débits observés montrent une variation de la salinité suivant les hauteurs d'eau: hauteur d'eau minimum (salinité faible) et hauteur d'eau maximale (salinité forte) (figure 4a). La turbidité présente des phases d'augmentation à chaque cycle de marée (morte et vive eau) (figures $4 \mathrm{a}$ et $4 \mathrm{~b}$ ). Les valeurs des pics de turbidité observées sont importantes en marée montante et moindre en marée descendante.
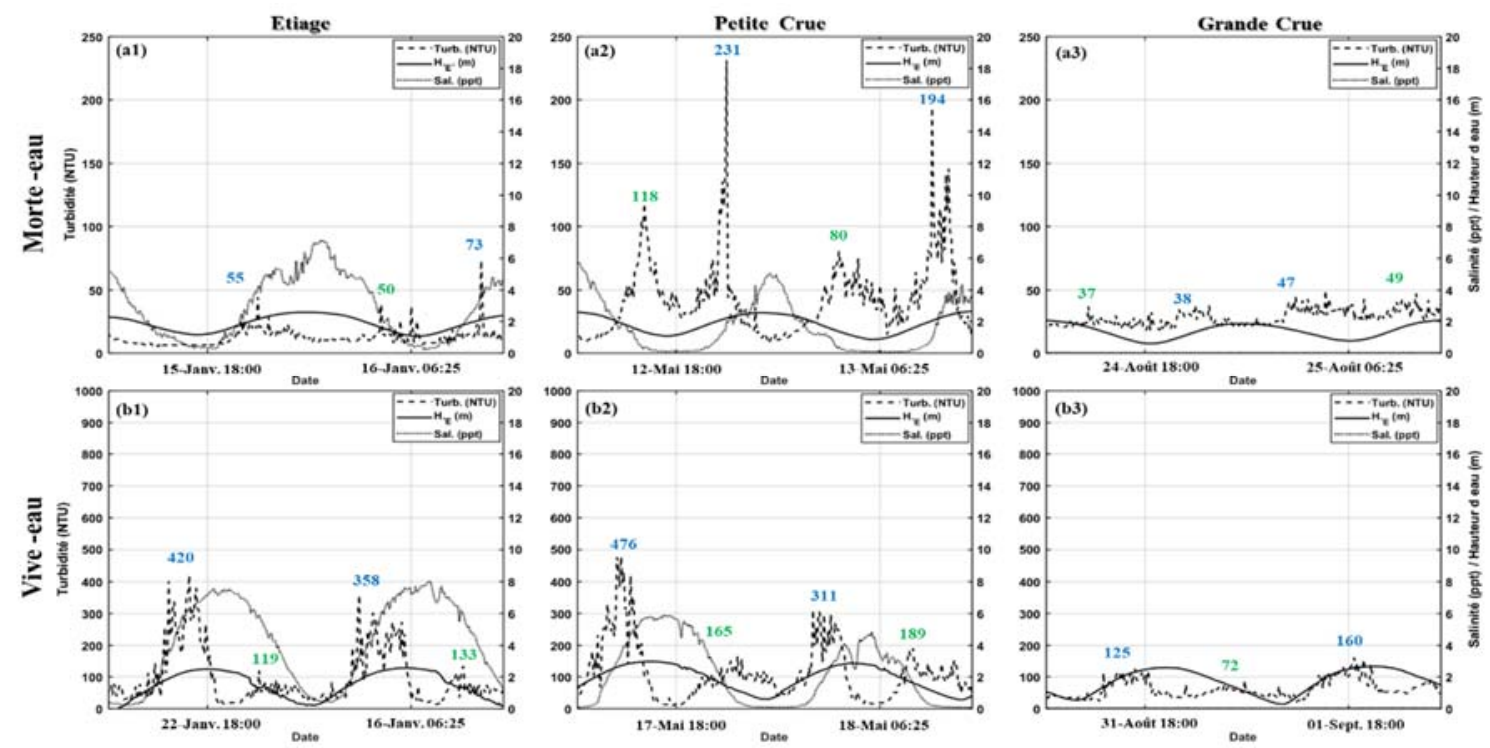

Figure 4. Variation de la Turbidité présentant ces valeurs maximales en marée montante (bleu) et descendante (verte), hauteur d'eau et salinité en fonction d'un cycle de marée observé en une journée pendant les mortes (a) et vives eaux (b) à la station de mesure amont de l'estuaire (noir) en période d'étiage (a1, b1), petite crue $(a 2, b 2)$ et grande crue (a3, b3). 


\section{XVİ̀mes Journées Nationales Génie Côtier - Génie Civil \\ Le Havre 2020}

\subsubsection{Station Aval (P.2)}

En aval, la salinité varie suivant les hauteurs d'eau (hauteur d'eau minimum (salinité faible) et hauteur d'eau maximale (salinité forte) comme en amont (figure 5a). Les variations de turbidité observées en période d'étiage et grande crue montre des phases d'augmentations à chaque cycle de marée (morte et vive eau). Inversement à l'observation faite en amont, les valeurs des pics de turbidité observées en marée montante sont moindres, comparées à celle observées en marée descendante. Les turbidités observées pendant la petite crue (figures $5 \mathrm{a} 2$ et $5 \mathrm{~b} 2$ ), montrent une non corrélation avec les variations de hauteurs d'eau présentant des valeurs élevées de turbidités (1500 $3800 \mathrm{NTU})$.
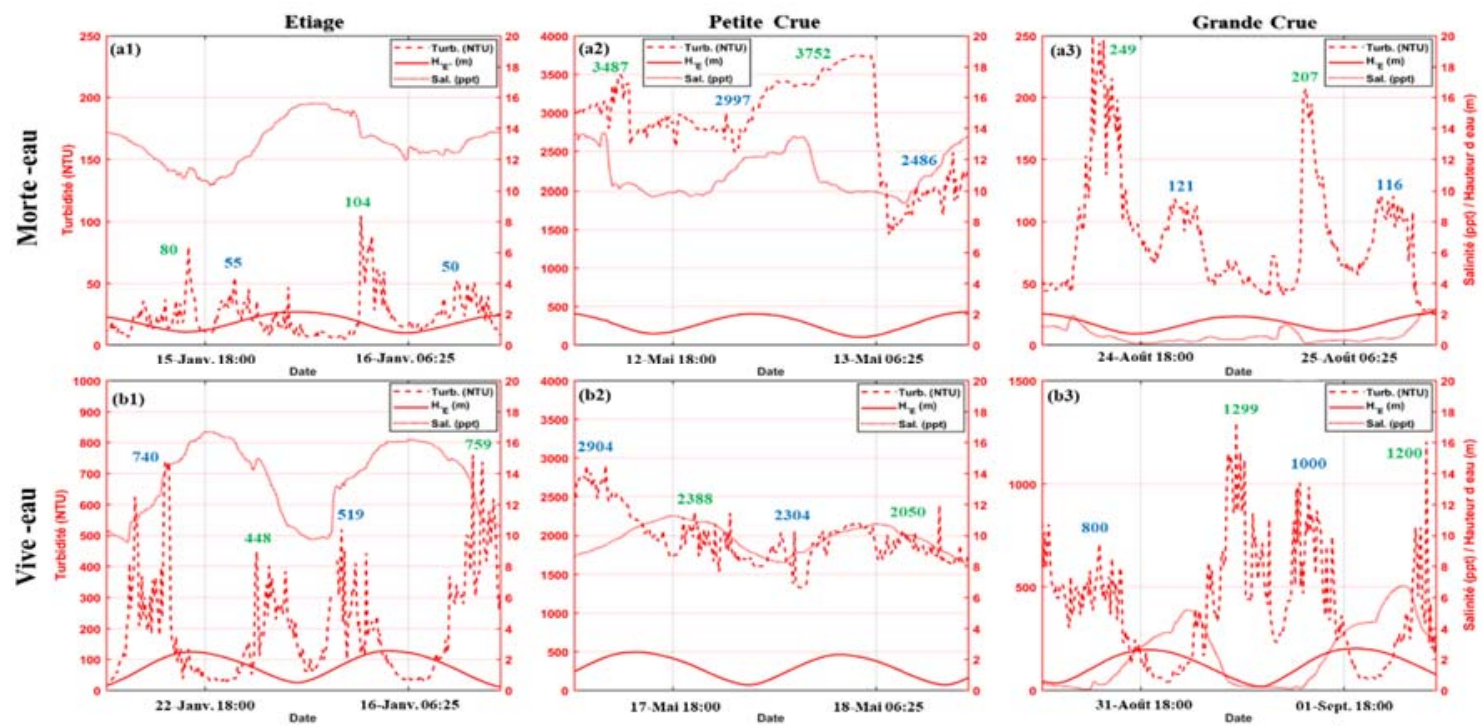

Figure 5. Variation de la Turbidité présentant ces valeurs maximales en marée descendante (bleu) et descendante (verte), hauteur d'eau et salinité en fonction d'un cycle de marée observé en une journée pendant les mortes (a) et vives eaux (b) à la station de mesures aval de l'estuaire (rouge) en période d'étiage (a1, b1), petite crue (a2, b2) et grande crue $(a 3, b 3)$.

4.3 Variations moyenne des maximums de turbidité en fonction des heures de marée Les valeurs de maximums moyenne de turbidité observées pendant les trois débits sont plus importantes en aval de l'estuaire (figure 6). En période d'étiage, le pic de turbidité est observé en marée montante (620 NTU) et en marée descendante pendant les crues (3764NTU et 2008 NTU, respectivement petite et grande crue) (figure 6b). 


\section{Thème 2 - Dynamique sédimentaire}
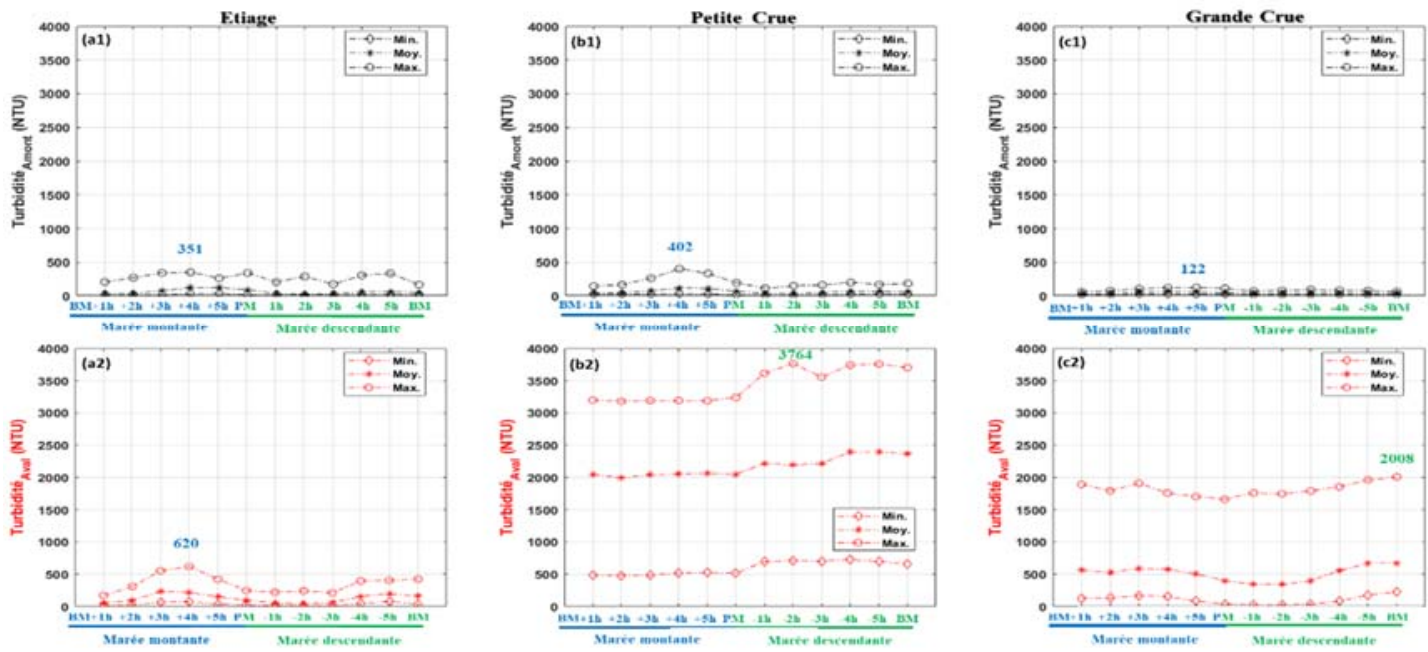

Figure 6. Variation de la turbidité, hauteur d'eau et salinité en fonction d'un cycle de marée observé en une journée pendant les mortes (a) et vives eaux (b) à la mesures aval de l'estuaire (rouge) en période d'étiage (a1, b1), petite crue (a2, b2) et grande crue $(a 3, b 3)$.

\section{Discussion-conclusion}

Le suivi et la localisation des zones de turbidité maximale (ZTM) est aujourd'hui un besoin dans l'estuaire du Wouri et d'un intérêt particulier pour améliorer la gestion régionale des sédiments. Le présent travail, basé sur des mesures de hauteurs d'eau, salinité et turbidité aux stations de mesures amont et aval au cours l'année 2019 révèle des informations détaillées sur la dynamique des sédiments en suspension pour des échelles de temps et de conditions hydrologiques différentes :

(a)- Variabilité en fonction des cycles de marée : Dans l'estuaire du Wouri, les fortes turbidités observées en vives eaux sont liées à l'intensité des courants maximums, qui est elle-même liée au marnage en vive eaux, (CORMAULT, 1971). À l'échelle semi-diurne, les turbulences générées par les courants de marée nous ont permis d’observer deux pics de turbidité :

En amont, en raison de la déformation de l'onde de marée dans l'estuaire, la durée du flot (marée montante) est plus courte que la durée du jusant (marée descendante) (FOSSI FOTSI, 2014). Ceci induit des vitesses de courant plus intenses en marée montante et se traduit par une grande capacité l'érosion du fond, expliquant la présence systématique du pic de turbidité maximale en présence le bouchon vaseux.

En aval, le pic de turbidité maximale observé en marée descendante (mi-jusant) peut être expliqué en première approche par l'existence d'une zone de forte dynamique, associée à l'érosion maximale, suggérée par ALLEN et al., 1980. Celle-ci résulte de la dissipation de la puissance de la marée, lors de sa propagation vers l'intérieur de l'estuaire (aval). La dissipation est maximale en aval, là où la convergence des rives impose à l'onde tidale 


\section{XVI'èmes Journées Nationales Génie Côtier - Génie Civil \\ Le Havre 2020}

une forte friction sur le fond. Cependant, il est plus vraisemblable que ce maximum soit la conséquence des remises en suspension locales de crème de vase.

Toutefois, les pics des pleines et basses mers, sont liés à des phénomènes locaux d'érosion et de dépôt, donc à la présence de crème de vase à proximité des stations amont et aval. (b)- Variabilité saisonnière: Le régime fluvial se matérialise par des migrations longitudinales de la position de la ZTM, et donc par le basculement amont-aval-amont ( MITCHELL, 2013). Les valeurs permanentes élevées de turbidité (1500 - 4000 NTU) montrent que la ZTM s'étend en aval de l'estuaire pendant la petite période de crue. Ceci peut être dû à plusieurs facteurs :

- le débit élevé du fleuve peut avoir apporté une grande quantité de sédiments de l'amont vers l'aval ;

- le débit élevé du fleuve a peut-être érodé la rive solide du fleuve, générant des sédiments frais qui se sont déposés et installés dans l'estuaire (SYVITSKI, 2005 ; UNCLES et al., 1994).

La morphologie de l'estuaire entre amont et aval induit une convergence localisant la ZTM entre les courants de marée montante orientés vers l'amont et ceux de marée descendante orientés vers l'aval. Plus le débit du fleuve est faible, plus les courants intenses de flot induisent une turbidité maximale essentiellement liée par la marée donc en migration vers l'amont. En période de crue, la ZTM est essentiellement liée au débit et donc expulsée par advection vers l'aval. Les valeurs de turbidité fortes en petite crue sont liées à la forte disponibilité des sédiments, largement déposés dans l'estuaire pendant l'étiage, et remis en suspension dès l'augmentation des débits fluviaux. Ce fait de variabilité saisonnière responsable du contrôle des changements de la position de la ZTM (amont et aval) montré est également observé dans les estuaires de la Loire, la Seine et la Gironde (GUEZENNEC et al., 1999 ; JALON-ROJAS et al., 2016).

Afin d'étayer nos hypothèses de compréhension des caractérisations des ZTM en estuaire mésotidal, une analyse des données des vitesses de courant et un modèle hydrodynamique pour simuler la turbidité sur l'ensemble de l'estuaire à différents régimes fluviaux et tidaux est en cours d'études. Ceci, nous permettra d'apprécier la variation verticale des fonds sur la circulation, la vitesse des courants et également d'estimer comment le transport par la marée et les débits influençant la ZTM et le contrôle les dépôts.

\section{Remerciements}

Les auteurs remercient le Service d'Action Culturel de l'Ambassade de France au Cameroun (SCAC), le laboratoire LIENS (CNRS/Université de La Rochelle) et le Shom (Brest) et Yves MIMBEU, doctorant de l'Université de Douala (Douala) pour son aide précieuse dans les campagnes de mesure. 


\section{Thème 2 - Dynamique sédimentaire}

\section{Références bibliographiques}

ALlEN G. P., SAlOMON J. C., BASSOUllet P., DU PENHOAT Y., DE GRANDPRE C. (1980). Effects of tides on mixing and suspended sediment transport in macrotidal estuaries. Sedimentary Geology, Vol. 26(1-3), pp 69-90. https://doi.org/10.1016/0037-0738(80)90006-8

AMELIE A. (2018). Analyse temporelle de la représentation du risque d'inondation de 1980 à 2018 à Douala-Cameroun. Rapport de Master, Université de Liège, 69 p.

CAPO S., SOTTOLICHIO A., BRENON I., CASTAING P., FERRY L. (2006). Morphology, hydrography and sediment dynamics in a mangrove estuary: The Konkoure Estuary, Guinea. Marine Geology, Vol. 230(3-4), pp 199-215. https://doi.org/10.1016/j.margeo.2006.05.003

CORMAULT P. (1971). Détermination expérimentale du débit solide d'érosion de sédiments fins cohésifs. $14{ }^{\text {ème }}$ Congrès de l'AIRH, Paris.

DIN N., SAENGER P., JULES P. R., SIEGFRIED D. D., BASCO F. (2008). Logging activities in mangrove forests: A case study of Douala Cameroon. African Journal of Environmental Science and Technology, Vol. 2(2), pp 22-30.

DJOMBE SEPPO D. (2011). Contribution à la connaissance de la dynamique sédimentaire du chenal d'accès au port de Douala. Rapport d'études, Port de Douala, $112 \mathrm{p}$.

ERECO EUROPEAN ECONOMIC. (1993). Etude de protection environnementale du port de Douala, du chenal d'accès et des eaux côtières (E2803 v1; 92 p.). Netherlands Economic Institute.

FOSSI FOTSI Y. (2014). Etude des paramètres hydrodynamiques de contrôle de la sédimentation dans l'estuaire du Wouri. Rapport de Master, Université de Douala, $51 \mathrm{p}$. FOSSI FOTSI Y., POUVREAU N., BRENON I., ONGUENE R., ETAME J. (2019). Temporal (1948-2012) and dynamic evolution of the Wouri estuary coastline within the gulf of Guinea. Journal of Marine Science and Engineering, Vol. 7(10), 343. https://doi.org/10.3390/jmse 7100343

GRABEMANN I., UNCLES R. J., KRAUSE G., STEPHENS J. A. (1997). Behaviour of turbidity maxima in the Tamar (U.K.) and Weser (F.R.G.), Estuaries. Estuarine, Coastal and Shelf Science, Vol. 45(2), pp 235-246. https://doi.org/10.1006/ecss.1996.0178

GUEZENNEC L., LAFITE R., DUPONT J.-P., MEYER R., BOUST, D. (1999). Hydrodynamics of suspended particulate matter in the tidal freshwater zone of a macrotidal estuary (The Seine estuary, France). Estuaries, Vol. 22(3), pp 717-727. https://doi.org/10.2307/1353058

JALÓN-ROJAS I., SCHMIDT S., SOTTOLICHIO A., BERTIER C. (2016). Tracking the turbidity maximum zone in the Loire Estuary (France) based on a long-term, highresolution and high-frequency monitoring network. Continental Shelf Research, Vol. 117, pp 1-11. https://doi.org/10.1016/j.csr.2016.01.017 


\section{XVİ̀mes Journées Nationales Génie Côtier - Génie Civil \\ Le Havre 2020}

MIGNIOT C. (1989). Manuel sur l'hydrodynamique sédimentaire et l'érosion et sédimentation du littoral. ER PM No 88.01, METL, CETMEF, SOGREAH-LCHF, 323 p. MITCHELL S., AKESSON L., UNCLES R. (2012). Observations of turbidity in the Thames Estuary, United Kingdom. Water and Environment Journal, Vol. 26(4), pp 511-520. https://doi.org/10.1111/j.1747-6593.2012.00311.x

MITCHELL S. B. (2013). Turbidity maxima in four macrotidal estuaries. Ocean \& coastal management, Vol. 79, pp 62-69. https://doi.org/10.1016/j.ocecoaman.2012.05.030

NDONGO B., MBOUENDEU S. L., TIRMOU A. A., NJILA R. N., DALlE J. D. M. (2015). Tendances pluviométriques et impact de la marée sur le drainage en zone d'estuaire: Cas du Wouri au Cameroun. Afrique Science: Revue Internationale des Sciences et Technologie, Vol. 11(2), pp 173-182.

OLIVRY J. C. (1974). Régime hydrologique du fleuve Wouri et estimation des apports reçus par l'estuaire et la mangrove du Wouri. Office de la recherche scientifique et technique Outre-Mer. $60 \mathrm{p}$.

SOGREAH. (2006). Etude du schéma directeur d'assainissement de la ville de Douala et maitrise d'œuvre d'une tranche prioritaire de travaux rapport définitif des phases 3 et 4, Rapport No 2350038 /DLN/FRB/PGN/ - avril 2006, 132 p.

SYVITSKI J. P. M. (2005). Impact of humans on the flux of terrestrial sediment to the global coastal ocean. Science, Vol. 308(5720), pp 376-380. https://doi.org/10.1126/science.1109454

TSALEFAC M., NGOUFO R., NKWAMBI W., DJOUMESSI TATSANGUE E., LENGUE FOBISSIE B. (2003). Fréquences et quantités des précipitations journalières sur le territoire camerounais. Publications de l'Association internationale de climatologie- AIC, Vol. 15, pp 359-367.

UNCLES R. J., BARTON M. L., STEPHENS, J. A. (1994). Seasonal variability of finesediment concentrations in the turbidity maximum region of the Tamar Estuary. Estuarine, Coastal and Shelf Science, Vol. 38(1), pp 19-39. https://doi.org/10.1006/ecss.1994.1002 
Thème 2 - Dynamique sédimentaire 